\title{
Retrospective Claims Analysis of Treatment Patterns, Relapse, Utilization, and Cost Among Patients with Multiple Sclerosis Initiating Second-Line Disease-Modifying Therapy
}

\author{
Leorah Freeman $^{1}$ (D) Arianna $\mathrm{Kee}^{2} \cdot \operatorname{MarC}^{\operatorname{Tian}^{2}} \cdot \mathrm{Rina} \mathrm{Mehta}^{2}$
}

Accepted: 19 April 2021 / Published online: 16 June 2021

(c) The Author(s) 2021

\begin{abstract}
Background Real-world studies of disease-modifying therapies (DMTs) in multiple sclerosis (MS) have reported suboptimal adherence.

Objective We aimed to describe treatment patterns, relapses, healthcare resource utilization, and costs in MS patients experiencing their first observed DMT switch.

Methods In this retrospective, claims database study, adult patients were selected if they had an MS diagnosis and DMT claim during the study period (1 January 2009-31 March 2019). Patients who switched to a new DMT between 1 January 2010 and 31 March 2018 were included. Adherence, persistence, relapses, and all-cause and MS-related healthcare utilization and costs were reported pre- and post-index.

Results In total, 1554 MS patients were identified; the mean age was 46 years and most (74\%) were female. The majority of patients switched from an injectable DMT $(n=1116 ; 71.8 \%)$, and patients generally switched to an oral DMT $(n=878 ; 57 \%)$. Among patients who switched DMTs, $46.0 \%(n=715)$ were nonadherent, $42 \%(n=645)$ were nonpersistent, and $21.5 \%$ $(n=334)$ relapsed in the 12 months post-switch. An increase in all-cause and MS-related healthcare costs was observed pre- to post-index for all patients. Cost drivers included outpatient visit costs and pharmacy prescriptions. Compared with patients who switched to an injectable DMT, those who switched to an oral DMT had significantly higher persistence and adherence. No significant difference was observed in post-index relapse or all-cause and MS-related total cost of care.

Conclusion Low adherence and poor persistence remain following an initial DMT switch; however, patients who switched to oral DMTs had higher persistence and adherence.
\end{abstract}

\section{Plain Language Summary}

Multiple sclerosis (MS) is a disabling disease that is treated with disease-modifying therapies (DMTs). Little is known about how patients with MS take their medication, how disease progression may change with treatment, or what the impact of switching to a new DMT is on the cost of care. In an analysis of commercially insured individuals, patients with MS were examined before and after switching to a new DMT. Results showed that the patients most often switched from an injectable medication to an oral DMT; however, a large proportion of patients did not take the prescription as directed by their physician. Additionally, a large proportion of patients did not stay on their new therapy. Nearly one-third of patients experienced an MS relapse after they switched to a new treatment, and healthcare costs increased following the treatment switch. A higher proportion of patients switching to an oral DMT took their medication as prescribed by their physicians, stayed on therapy, and incurred smaller increases in cost compared with patients switching to injectable medications. Despite such improvements, additional treatments are needed for patients with MS.

Leorah Freeman

leorah.freeman@austin.utexas.edu

1 Health Discovery Building, Dell Medical School, The University of Texas at Austin, 1601 Trinity St, Austin, TX 78701, USA

2 Bristol Myers Squibb, Princeton, NJ, USA 


\section{Key Points}

Overall, treatment adherence and persistence were low among MS patients who switched to a second DMT, and nearly one-third of patients relapsed within 12 months following the switch; all-cause and MS-related costs increased following the DMT switch.

Patients switching to an oral DMT had significantly higher treatment adherence and persistence compared with patients switching to an injectable DMT.

Although improvements in treatment measures and relapses were observed after switching to a second DMT, the incidence of non-adherence, non-persistence, and relapse remain high and indicate the need for improved treatment options.

\section{Introduction}

Multiple sclerosis (MS) is a chronic autoimmune disease of the central nervous system (CNS) characterized by inflammatory and neurodegenerative processes $[1,2]$. The prevalence of MS in the USA, estimated at 900,000 individuals in 2017 , has been increasing steadily, a trend thought to arise from an aging population with improved survival [3]. The clinical manifestations of MS include vision disturbance and loss, fatigue, weakness, gait instability, bladder dysfunction, and cognitive deficits [4]. The disease most commonly presents in alternating relapses and remissions in neurologic dysfunction, as in relapsing-remitting MS (RRMS), and relapses may be associated with development of long-term disability and a poorer prognosis [4-6].

The economic and societal burden of MS is high owing to increasing healthcare costs, caregiving costs, and productivity losses; for patients, MS lowers quality of life (QoL) and leads to high levels of disability [4, 7]. Greater frequency and severity of MS relapses, increased overall disease severity, and disability progression are all significantly associated with a higher cost burden [4, 8, 9].

MS guidelines recommend that patients with RRMS be treated with disease-modifying therapies (DMTs), which can reduce the rate of relapse, slow disease progression, and lead to better long-term outcomes $[1,10,11]$. The choice of DMT is influenced by a variety of factors-mechanisms of action, efficacy, dosing schedules, routes of administration, tolerability, and safety-which differ by DMT [12]. Available DMTs for MS consist of injectable, oral, and infusion therapies across nine drug classes [13]. Injectables were the first DMTs approved in the 1990s and include the immune modulators interferon beta- $1 \mathrm{~b}$ and glatiramer acetate for treatment for RRMS. In 2004, the first infusion therapeutic, natalizumab, was approved, and since then, other immune suppressant infusions such as alemtuzumab and ocrelizumab have been approved. The first oral DMT, fingolimod, was approved in 2010 and, as of April 2020, there were six additional approved oral treatments for MS, including cladribine, dimethyl fumarate, diroximel fumarate, ozanimod, teriflunomide, and siponimod [13]. Adherence and persistence to DMT regimens are of critical importance in achieving full efficacy; however, real-world studies of DMT treatment patterns in MS have reported suboptimal adherence rates $[10,12]$. When making treatment decisions, clinicians may be advised to address potential barriers to adherence, such as cognitive impairment and depression, perceived lack of efficacy, and adverse events [10, 14]. Nonadherence to DMT regimens has been associated with an increased risk of relapse, poorer health outcomes, and higher healthcare resource utilization (HCRU) among patients with MS [1, 14]. Strategies to improve adherence may lead to better outcomes in MS, as patients who are adherent to their treatment regimens have lower relapse rates, reduced HCRU, and lower healthcare costs than nonadherent patients [10, 12].

Initial choice of treatment and subsequent treatment modifications are often driven by the risk of disease progression. Escalation - an approach to therapy that begins with less potent and relatively safer DMTs-is most often used for patients with MS [2, 15, 16]. Switches between DMTs are considered after breakthrough disease activity (evidence of continued relapses or activity on magnetic resonance imaging). MS guidelines recommend that clinicians also factor in patient preference, tolerability, and likelihood of adherence when selecting a subsequent DMT [10].

Several studies have examined recent real-world treatment patterns, HCRU, and cost among patients with MS treated with DMTs [1, 15, 17-19]. Given the speed at which the treatment landscape is evolving [20], it is important that we evaluate treatment patterns with the most current DMTs. Furthermore, there is a need to clarify the experience of patients who have switched to a second DMT [15]. Currently, few studies have examined the real-world use of second-line DMTs. One recent study found that patients who switched to second-line MS treatment had approximately $50 \%$ persistence and adherence with a proportion $(29 \%)$ of patients still experiencing a relapse, highlighting the need for additional treatment options [15]. The objective of this study was to describe treatment patterns, relapses, healthcare costs, and HCRU in a MS patient population experiencing their first DMT treatment switch recorded in a large claims database stratified by DMT route of administration. We hypothesize that route of administration of new therapy 
following a DMT switch impacts adherence, persistence, and patient outcomes in MS.

\section{Methods}

\subsection{Study Design and Data Source}

This retrospective, observational cohort study was conducted using administrative claims data contained in the Optum Clinformatics ${ }^{\mathrm{TM}}$ Data Mart database in the USA (OptumInsight, Inc., Eden Prairie, MN, USA). The Clinformatics Data Mart database includes data from approximately 15-18 million lives with annual healthcare coverage. Data for both commercial health plan and Medicare Advantage members are included in this database.

Optum's standard pricing algorithms were used to obtain prices for each service provider to reflect the intensity of care. Standard pricing reflected quantity of services provided, relative resource costs, and healthcare utilization type. Facility inpatient pricing, estimated as cost per diem, was based on aggregated diagnostic categories and length of stay. Facility outpatient pricing was determined from revenue codes and the reimbursement requested from the provider. Pharmacy costs were based on First Databank pricing and adjusted according to therapeutic category and generic indicator. Professional and ancillary service pricing was estimated using Centers for Medicare and Medicaid Services relative value units.

\subsection{Multiple Sclerosis Patient Selection and Cohort Assignment}

The full study period (1 January 2009-31 March 2019) consisted of a 12-month pre-index (baseline) period, a patientselection period, and a 12-month post-index (evaluation) period (Fig. 1). Adults (age $\geq 18$ years on the date of a first switch to a new DMT) were selected for inclusion during the patient selection period if they had evidence of an MS diagnosis, defined as two nondiagnostic service claims (1-365 days apart) for MS (ICD-9 code 340 [1 January 2009-30 September 2015] or ICD-10 code G35 [1 October 2015-31 March 2018]) and a claim for an oral, injectable, or infusion DMT (initial DMT) during the study period [21]. Eligible patients who switched to a new DMT between 1 January 2010 and 31 March 2018 were included in the study, and the date of switch was their index date. Continuous healthcare plan enrollment was required for the 12 months pre-index and 12 months post-index date. Patients were excluded if there was evidence of any of the following: pregnancy or malignancy during the 12-month pre- and post-index periods or known use of any DMT prior to the date of starting the initial DMT.

\subsection{Outcomes}

Clinical characteristics, DMT treatment patterns (adherence and persistence), algorithm-based relapse rates, healthcare costs, and HCRU were described. Adherence was measured as the proportion of days covered (PDC) with the index DMT during the 12-month post-index period, and the adherence threshold was established as 0.8 . Persistence on the index DMT was defined as the number of days from the index date until the earliest of either treatment discontinuation (>60-day gap in therapy after current supply ended) or a switch to a new DMT. Patients who restarted their index DMT or switched to a new DMT after meeting discontinuation criteria were captured, as was the time to restart or switch (i.e., date of discontinuation to date of restart or switch).

Relapse was identified via a validated claims-based algorithm [22, 23] as the presence of any of the following: (1) MS-related inpatient stay (an inpatient admission with ICD9-CM 340 or ICD-10-CM G35 as the primary diagnosis) or (2) an outpatient claim with an MS diagnosis code in the primary or secondary position and a pharmacy or medical claim for a qualifying corticosteroid or adrenocorticotropic hormone on the day of or within 7 days after the outpatient visit. The date of the relapse was recorded as the date on a medical claim identifying one of these two criteria.

All-cause and MS-related healthcare costs were recorded for all patients and among those with at least one service for the 12-month pre-index (baseline) and 12-month postindex (evaluation) periods. All estimates were inflated to 2018 dollars using the Medical Care Component of the Consumer Price Index. Mean healthcare costs were reported as total expenditures and by category for inpatient, emergency department (ED), and outpatient visits and pharmacy costs.

All-cause and MS-related HCRU were recorded for all patients for the 12-month pre-index and 12-month postindex periods. HCRU outcomes for hospital admissions included percentage of patients with inpatient admissions, number of inpatient admissions, and the average length of stay (LOS). ED visits were recorded as the number of ED visits for the overall sample and the percentage of patients with any ED visits. Outpatient office visits were recorded as the percentage of patients with an office visit with a neurologist, primary-care provider, or other provider. Outpatient prescriptions were also recorded. 
Fig. 1 Study flow. DMT disease-modifying therapy, $M S$ multiple sclerosis

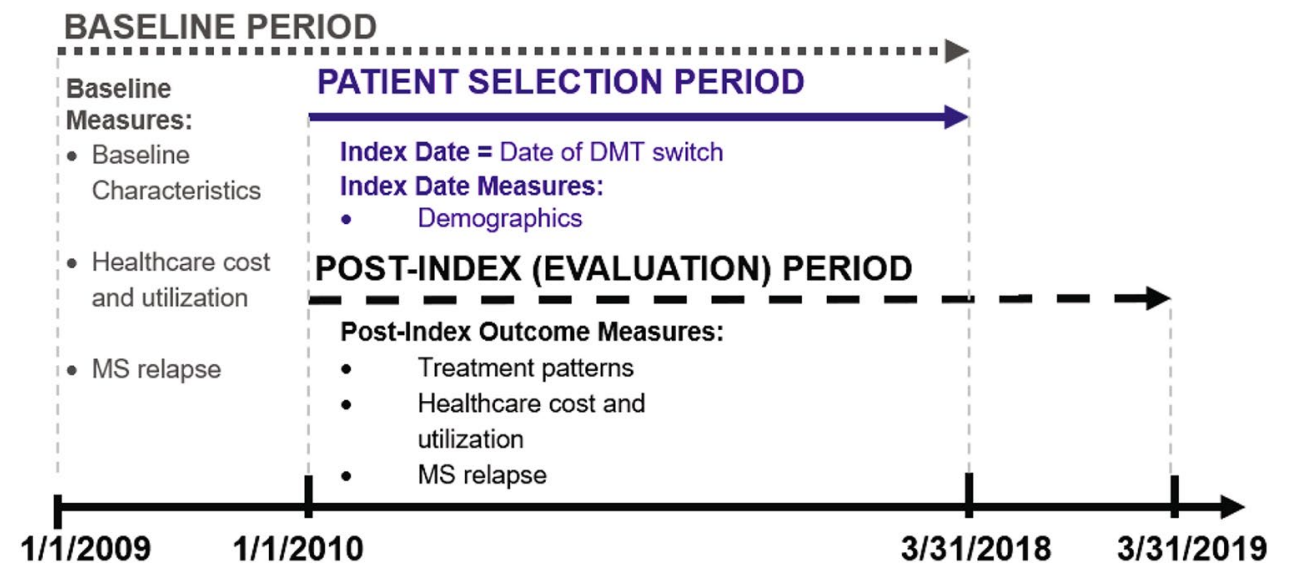

\subsection{Statistical Analysis}

Bivariate analysis for all variables was conducted, and patients were stratified by DMT route of administration. Infusion DMTs included alemtuzumab, mitoxantrone, natalizumab, and ocrelizumab; injectable DMTs included glatiramer acetate, interferon beta-1a, interferon beta- $1 \mathrm{~b}$, and peginterferon beta-1a; and oral DMTs included dimethyl fumarate, fingolimod, and teriflunomide. Categorical variables were reported as the count and percentage of patients, and continuous variables were reported as the mean, standard deviation, and median (when appropriate). A multivariate analysis was conducted using logistic regression to examine the odds of post-index adherence, persistence, and relapse. A Gamma model with log link function was used to examine post-index all-cause and MS-related healthcare costs. All models were adjusted for select patient characteristics that included the initial DMT route of administration, time from end of initial DMT to index date, number of baseline relapses, age (by decade), sex, index year, geographic region of residence, select comorbid conditions (depression, hyperlipidemia, hypertension, fatigue, and neuropathic pain), and baseline medications (antidepressants, antispasmodics, nonsteroidal anti-inflammatory drugs [NSAIDs] or cyclooxygenase 2 [COX-2] inhibitors, neuropathic pain medications, and opioids). For cost models, we controlled for pre-index all-cause healthcare costs. Subgroup analyses included data from patients who initiated an injectable DMT and switched to another discrete DMT (either oral, injectable, or infusion), as these individuals composed the majority of the study population.

All data programming and statistical analyses were performed using SAS software (SAS Institute, Cary, NC, USA).

\section{Results}

\subsection{Baseline Characteristics}

Of 100,845 patients with MS, 1554 (1.5\%) met the study inclusion criteria and were included in the final sample (Fig. 2). Patients had a mean age of 46 years at the index date (DMT switch), and the majority of patients were female $(73.6 \%)$ (Table 1). Demographic characteristics for the overall population and by index DMT generally were consistent across routes of administration (oral, injectable, and infusion), with the exception of year, which varied because of differences in regulatory approval of the individual agents. The majority of patients were initially treated with injectable DMTs (71.8\%); fewer patients initially received oral (22.4\%) and infusion (5.8\%) DMTs. Clinical characteristics were also analyzed for the overall population and by index DMT (Table 2). Fatigue was the most commonly reported comorbidity in the overall population, and a greater percentage of patients with index infusion DMTs were reported to have gait and mobility difficulties.

\subsection{Treatment Patterns}

At the time of the switch (index date), patients were primarily initiated on oral $(n=878$ [56.5\%]), followed by injectable $(n=394$ [25.4\%]) and infusion $(n=282$ [18.2\%]), DMTs. The most common index DMTs according to route of administration were dimethyl fumarate $(30.3 \%$, oral), glatiramer acetate (14.4\%, injectable), and natalizumab (12.9\%, infusion) (Fig. S1 in Online Supplemental Material [OSM]). Across all routes of administration, the mean time between initial and index DMTs was less than 5 months. Treatment patterns of concomitant medication use in the post-index period showed that opioids were most commonly prescribed (40\%), followed by NSAID/COX-2 inhibitors (28\%) and benzodiazepines $(25 \%)$ (Table 2). 
Overall, $46.0 \%$ of the population was nonadherent, with a mean (SD) PDC of 0.7 (0.31) over the 12-month post-index period. Patients who switched to injectable DMTs had the lowest rate of adherence (41.9\%) compared with those who switched to oral (54.1\%) and infusion (70.6\%) DMTs (Fig. S2A in OSM).

Consistent with the results for nonadherence, $41.5 \%$ of the population was nonpersistent. Patients who switched to injectable DMTs had the lowest rate of persistence $(44.4 \%)$ compared with those who switched to oral (60.5\%) and infusion (72.0\%) DMTs (Fig. S2B in OSM). The mean time to nonpersistence was less than 5 months after the switch. Among the nonpersistent patients, 90.2\% discontinued treatment, 9.8\% switched to a different DMT, and $19.8 \%$ of patients who discontinued their treatment restarted their index DMT.

\subsection{Relapse Rates}

More than $20 \%$ of patients relapsed in the 12 -month postindex period (Fig. S3 in OSM). In the overall population, the proportion of relapse-free patients increased from pre- to post-index, from 67.6 to $78.5 \%$. Additionally, relapse rates decreased from 29.4 to $20.3 \%$ among oral DMT users, 33.8 to $24.1 \%$ among injectable DMT users, and 39.7 to $21.6 \%$ among infusion DMT users in the 12 months post-index. The percentage reduction in relapse rate was slightly higher among patients who switched to oral DMTs (31\%) compared with those who switched to injectable DMTs (29\%). Patients who experienced a relapse in the pre-index period were more likely to experience a relapse in the post-index period; $85.5 \%$ of oral DMT users with no pre-index relapse were relapse-free in the post-index period compared with $71.6 \%, 52.1 \%$, and $45.0 \%$ of patients who had one, two, and three pre-index relapses, respectively. Similar trends were observed for the injectable and infusion DMT users (Table S1 in OSM). Among patients with two pre-index
Fig. 2 Patient disposition. $D M T$ disease-modifying therapy, $I C D-9-C M / I C D-10-C M$ International Classification of Diseases, Ninth/Tenth Revision, Clinical Modification, $M S$ multiple sclerosis

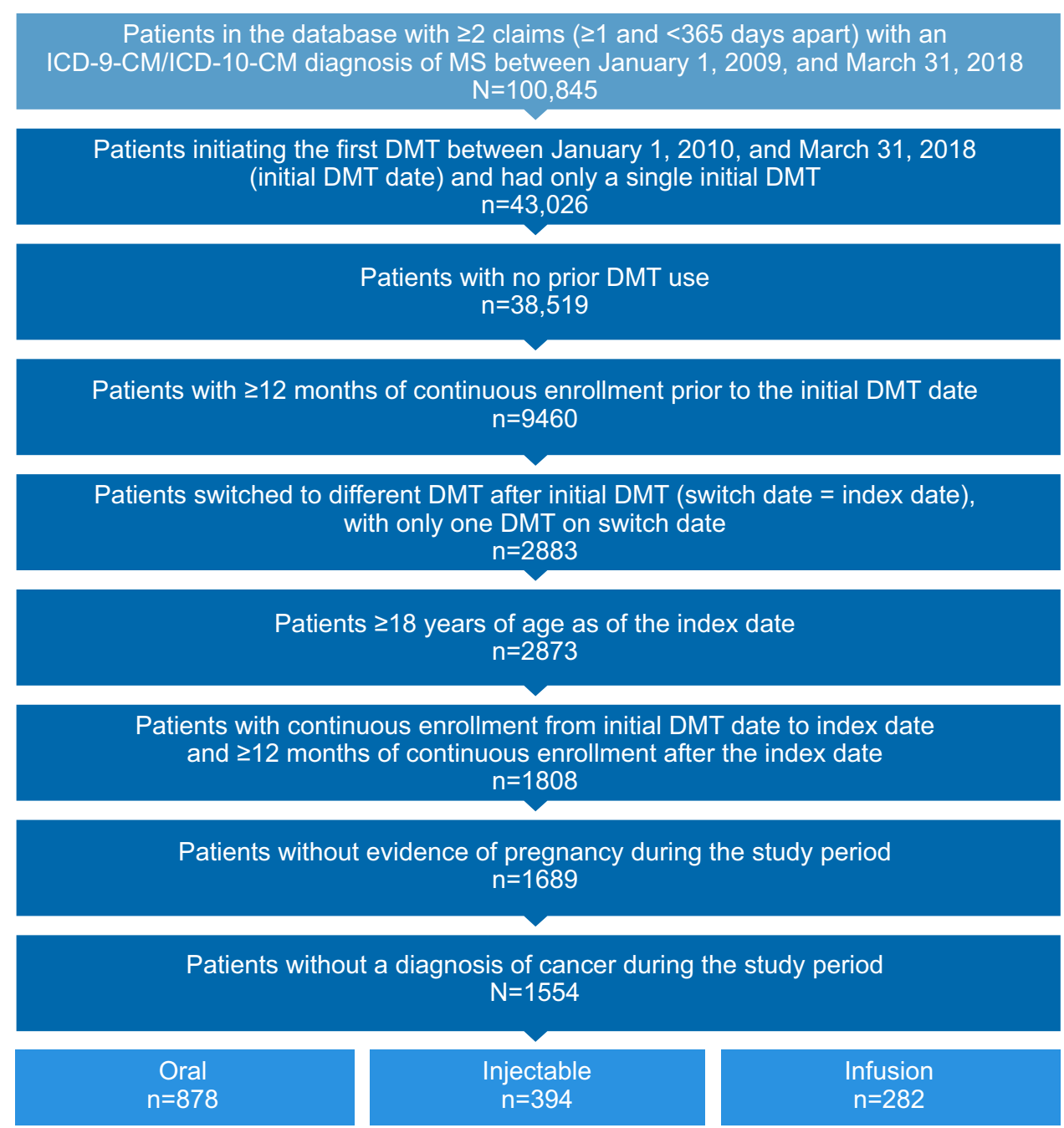


Table 1 Baseline demographic characteristics

\begin{tabular}{|c|c|c|c|c|}
\hline & All patients $(N=1554)$ & Oral $(n=878)$ & Injectable $(n=394)$ & Infusion $(n=282)$ \\
\hline Age, mean (SD), years & $46(12)$ & $47(12)$ & $46(12)$ & $45(13)$ \\
\hline \multicolumn{5}{|l|}{ Sex, $n(\%)$} \\
\hline Male & $411(26.4)$ & $233(26.5)$ & $102(25.9)$ & $76(27.0)$ \\
\hline Female & $1143(73.6)$ & $645(73.5)$ & $292(74.1)$ & $206(73.0)$ \\
\hline \multicolumn{5}{|l|}{ Race, $n(\%)$} \\
\hline Asian & $19(1.2)$ & $9(1.0)$ & $6(1.5)$ & $4(1.4)$ \\
\hline Black & $178(11.5)$ & $102(11.6)$ & $48(12.2)$ & $28(9.9)$ \\
\hline Hispanic & $112(7.2)$ & $71(8.1)$ & $24(6.1)$ & $17(6.0)$ \\
\hline White & $1115(71.8)$ & $614(69.9)$ & $287(72.8)$ & $214(75.9)$ \\
\hline Unknown & $130(8.4)$ & $82(9.3)$ & $29(7.4)$ & $19(6.7)$ \\
\hline \multicolumn{5}{|l|}{ Region, $n(\%)$} \\
\hline Northeast & $172(11.1)$ & $89(10.1)$ & $51(12.9)$ & $32(11.3)$ \\
\hline South & $615(39.6)$ & $343(39.1)$ & $158(40.1)$ & $114(40.4)$ \\
\hline Midwest & $385(24.8)$ & $217(24.7)$ & $99(25.1)$ & $69(24.5)$ \\
\hline West & $379(24.4)$ & $228(26.0)$ & $86(21.8)$ & $65(23.0)$ \\
\hline \multicolumn{5}{|l|}{ Payer, $n(\%)$} \\
\hline Commercial & $1169(75.2)$ & $648(73.8)$ & $297(75.4)$ & $224(79.4)$ \\
\hline Medicare & $385(24.8)$ & $230(26.2)$ & 97 (24.6) & $58(20.6)$ \\
\hline \multicolumn{5}{|l|}{ Index year, $n(\%)$} \\
\hline 2010 & $52(3.3)$ & $7(0.8)$ & $41(10.4)$ & $4(1.4)$ \\
\hline 2011 & $110(7.1)$ & $29(3.3)$ & $51(12.9)$ & $30(10.6)$ \\
\hline 2012 & $137(8.8)$ & $27(3.1)$ & $71(18.0)$ & $39(13.8)$ \\
\hline 2013 & $272(17.5)$ & $221(25.2)$ & $34(8.6)$ & $17(6.0)$ \\
\hline 2014 & $251(16.2)$ & $188(21.4)$ & $46(11.7)$ & $17(6.0)$ \\
\hline 2015 & $229(14.7)$ & $144(16.4)$ & $57(14.5)$ & $28(9.9)$ \\
\hline 2016 & $205(13.2)$ & $119(13.6)$ & $44(11.2)$ & $42(14.9)$ \\
\hline 2017 & $218(14.0)$ & $122(13.9)$ & $42(10.7)$ & $54(19.1)$ \\
\hline 2018 & $80(5.1)$ & $21(2.4)$ & $8(2.0)$ & $51(18.1)$ \\
\hline
\end{tabular}

relapses, a higher proportion who switched to oral DMTs $(52.1 \%)$ were relapse-free in the post-index period compared with those who switched to injectable $(48.0 \%)$ or infusion $(50.0 \%)$ DMTs; results were similar among those with three pre-index relapses (Table $\mathrm{S} 1$ in OSM).

\subsection{Healthcare Costs}

Mean all-cause and MS-related healthcare costs are presented in Table 3. While all-cause pharmacy costs were similar in the post-index period across all routes of administration (oral: $\$ 62,021$; injectable: $\$ 61,527$; and infusion: $\$ 62,299)$, all-cause total cost of care was higher among patients who switched to infusion DMTs $(\$ 107,610)$ than among those who switched to injectable $(\$ 76,076)$ and oral $(\$ 75,589)$ DMTs. Furthermore, patients who switched to infusion DMTs had the greatest increase in all-cause total cost of care from pre- to post-index $(\$ 38,325)$, followed by those who switched to injectable $(\$ 24,177)$ and oral $(\$ 21,696)$ DMTs. All costs are reported in US dollars.

The trend was similar for MS-related healthcare costs, with total cost of care highest among patients who switched to infusion DMTs $(\$ 84,551)$, followed by injectable $(\$ 68,867)$ and oral $(\$ 64,696)$ DMTs. Furthermore, patients who switched to infusion DMTs had the greatest increase in MS-related total cost of care from pre- to post-index $(\$ 28,993)$, followed by those who switched to injectable $(\$ 26,177)$ and oral $(\$ 19,655)$ DMTs.

\subsection{Healthcare Resource Utilization}

Analysis of all-cause HCRU showed a decrease in all-cause medical utilization overall (with the exception of outpatient office visits) from pre- to post-index across all routes of administration (Table 4). There was a notable increase 
Table 2 Clinical characteristics and concomitant medications

\begin{tabular}{|c|c|c|c|c|}
\hline & $\begin{array}{l}\text { All patients } \\
(N=1554)\end{array}$ & Oral $(n=878)$ & Injectable $(n=394)$ & Infusion $(n=282)$ \\
\hline \multicolumn{5}{|l|}{ Comorbidity at index, $n(\%)$} \\
\hline Bladder dysfunction & $346(22.3)$ & $197(22.4)$ & $82(20.8)$ & $67(23.8)$ \\
\hline Bowel dysfunction & $194(12.5)$ & $117(13.3)$ & $49(12.4)$ & $28(9.9)$ \\
\hline Depression & $395(25.4)$ & $225(25.6)$ & $94(23.8)$ & $76(27.0)$ \\
\hline Diabetes & $188(12.1)$ & $101(11.5)$ & $53(13.5)$ & $34(12.1)$ \\
\hline Dizziness and vertigo & $237(15.3)$ & $120(13.7)$ & $67(17.0)$ & $50(17.7)$ \\
\hline Fatigue & $558(35.9)$ & $303(34.5)$ & $147(37.3)$ & $108(38.3)$ \\
\hline Gait and mobility difficulties & 403 (25.9) & $214(24.4)$ & $93(23.6)$ & $96(34.0)$ \\
\hline Gastrointestinal disease & $223(14.4)$ & $121(13.8)$ & $60(15.2)$ & $42(14.9)$ \\
\hline Hyperlipidemia & $428(27.5)$ & $250(28.5)$ & 105 (26.6) & $73(25.9)$ \\
\hline Hypertension & $477(30.7)$ & $274(31.2)$ & $133(33.8)$ & $70(24.8)$ \\
\hline Neuropathic pain & $276(17.8)$ & $160(18.2)$ & $73(18.5)$ & $43(15.2)$ \\
\hline Other chronic pain & $161(10.4)$ & $95(10.8)$ & $38(9.6)$ & $28(9.9)$ \\
\hline Pruritus & $25(1.6)$ & $15(1.7)$ & $7(1.8)$ & $3(1.1)$ \\
\hline Pseudobulbar affect & $5(0.3)$ & $4(0.5)$ & - & $1(0.4)$ \\
\hline Sexual problems & $43(2.8)$ & $26(3.0)$ & $7(1.8)$ & $10(3.5)$ \\
\hline Spasticity & $108(6.9)$ & $56(6.4)$ & $25(6.3)$ & $27(9.6)$ \\
\hline Tremors & $29(1.9)$ & $18(2.1)$ & $3(0.8)$ & $8(2.8)$ \\
\hline Urinary tract infection & $274(17.6)$ & $156(17.8)$ & $72(18.3)$ & $46(16.3)$ \\
\hline \multicolumn{5}{|c|}{ Concomitant medications post-index, $n(\%)$} \\
\hline Antidiabetics & $135(8.7)$ & $72(8.2)$ & $35(8.9)$ & $28(9.9)$ \\
\hline Antihypertensives & $390(25.1)$ & $234(26.7)$ & $100(25.4)$ & $56(19.9)$ \\
\hline Benzodiazepines & $388(25.0)$ & $227(25.9)$ & $93(23.6)$ & $68(24.1)$ \\
\hline Antihyperlipidemics & $302(19.4)$ & $193(22.0)$ & $69(17.5)$ & $40(14.2)$ \\
\hline NSAID/COX-2 inhibitors & $441(28.4)$ & $251(28.6)$ & $111(28.2)$ & 79 (28.0) \\
\hline Opioids & $621(40.0)$ & $356(40.5)$ & $155(39.3)$ & $110(39.0)$ \\
\hline Dalfampridine & $138(8.9)$ & $79(9.0)$ & $23(5.8)$ & $36(12.8)$ \\
\hline
\end{tabular}

COX-2 cyclooxygenase $2, N S A I D$ nonsteroidal anti-inflammatory drug in mean (SD) inpatient LOS among patients who switched to infusion DMTs from 12.1 (17.2) days in the pre-index to 20.8 (38.3) days in the post-index period. A decrease in MS-related medical utilization (proportion of patients with inpatient admissions) was also observed from pre- to postindex across all routes of administration. As with all-cause HCRU, change in mean (SD) MS-related inpatient LOS was greater among patients who switched to infusion DMTs at 8.6 (10.6) days in the pre-index period compared with 26.3 (26.9) days in the post-index period.

\subsection{Multivariate Analyses}

The multivariate analyses showed that patients who switched to oral (odds ratio [OR]: $1.84 ; 95 \%$ confidence interval [CI] 1.39-2.43; $p<0.0001$ ) or infusion (OR: 3.77; 95\% CI 2.64-5.40; $p<0.0001)$ DMTs had significantly higher adherence rates compared with patients who switched to injectable DMTs (reference). Likewise, persistence was also significantly higher for patients switching to oral (OR: 2.03; 95\% CI 1.54-2.67; $p<0.0001$ ) or infusion (OR: 3.62; 95\% CI 2.53-5.19; $p<0.0001)$ DMTs than for those switching to injectable DMTs. There was no significant difference in post-index relapse rates between patients who switched to injectable DMTs and those who switched to oral (OR: 0.85 ; 95\% CI $0.61-1.20 ; p=0.3682$ ) or infusion (OR: $0.75 ; 95 \%$ CI $0.49-1.14 ; p=0.1765)$ DMTs. However, each additional pre-index relapse was associated with a $96 \%$ increase in odds of a post-index relapse $(p<0.0001)$. Compared with patients who switched to injectable DMTs, patients who switched to infusion DMTs had significantly increased all-cause (OR: 1.27; 95\% CI 1.18-1.38; $p<0.0001)$ and MS-related (OR: $1.19 ; 95 \%$ CI $1.08-1.31 ; p=0.0004)$ total cost of care. In contrast, there were no significant differences between those who switched to oral versus injectable DMTs for allcause total cost of care (OR: $1.00 ; 95 \%$ CI 0.94-1.107; $p=$ 0.8820 ) or MS-related total cost of care (OR: 0.99; $95 \% \mathrm{CI}$ $0.91-1.07 ; p=0.7926)$. 


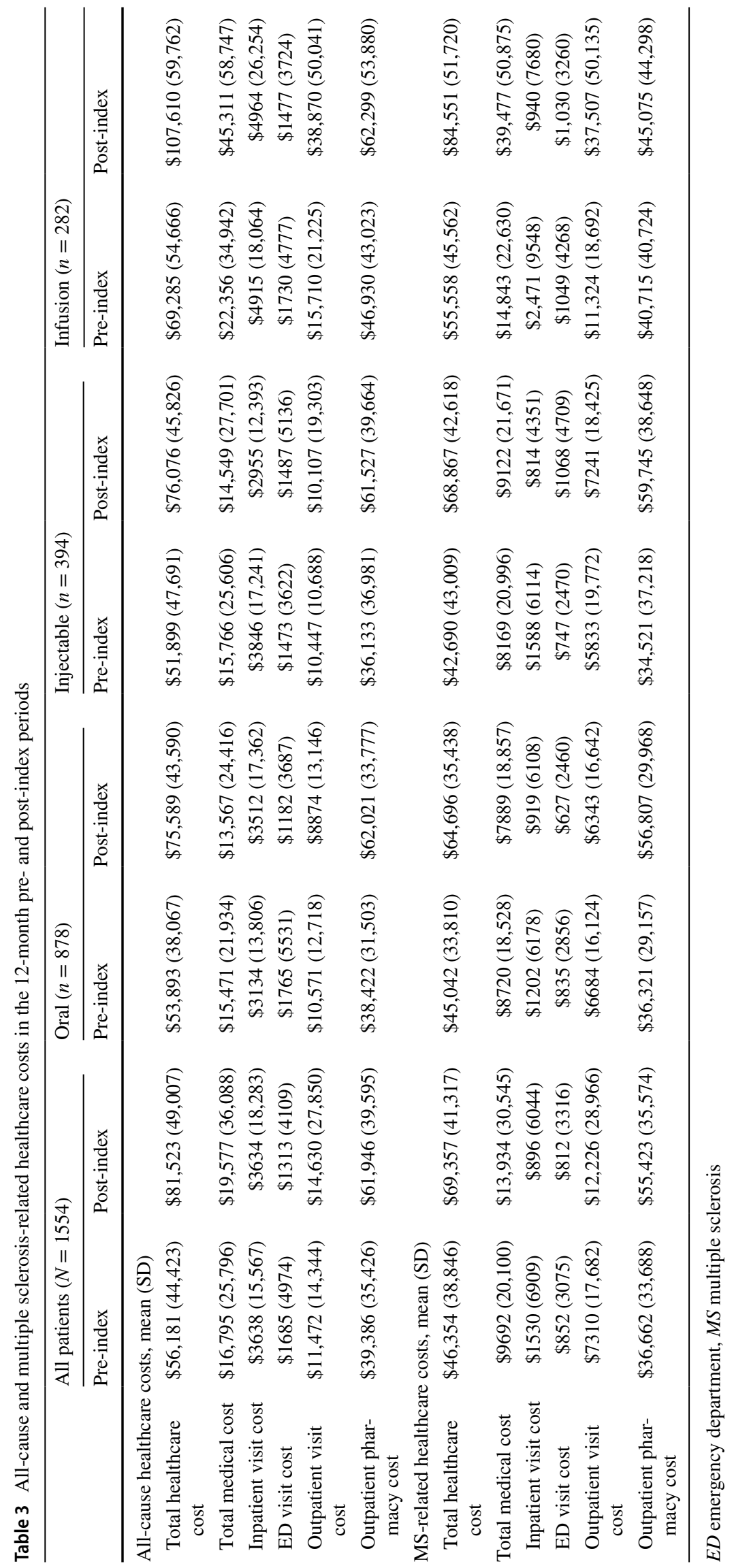


Table 4 All-cause and multiple sclerosis-related healthcare utilization in the 12-month pre- and post-index periods

\begin{tabular}{|c|c|c|c|c|c|c|c|c|}
\hline & \multicolumn{2}{|c|}{ All patients $(N=1554)$} & \multicolumn{2}{|l|}{ Oral $(\mathrm{n}=878)$} & \multicolumn{2}{|c|}{ Injectable $(n=394)$} & \multicolumn{2}{|c|}{ Infusion $(n=282)$} \\
\hline & Pre-index & Post-index & Pre-index & Post-index & Pre-index & Post-index & Pre-index & Post-index \\
\hline \multicolumn{9}{|l|}{ All-cause HCRU } \\
\hline $\begin{array}{l}\text { Inpatient admission, } \\
n(\%)\end{array}$ & $233(15.0)$ & 181 (11.6) & $112(12.7)$ & $98(11.2)$ & $74(18.8)$ & $46(11.68)$ & $47(16.7)$ & $37(13.1)$ \\
\hline LOS, mean (SD) & $10.34(20.55)$ & $14.2(33.76)$ & $11.75(25.78)$ & $14(38.56)$ & $7.09(11.38)$ & $9.33(10.97)$ & $12.11(17.28)$ & $20.81(38.28)$ \\
\hline $\begin{array}{l}\text { Patients with ED } \\
\text { visit, } n(\%)\end{array}$ & $525(33.8)$ & $457(29.4)$ & $284(32.3)$ & $260(29.6)$ & $143(36.3)$ & $108(27.4)$ & $98(34.8)$ & 89 (31.6) \\
\hline $\begin{array}{l}\text { Number of ED visits, } \\
\text { mean (SD) }\end{array}$ & $0.65(1.38)$ & $0.55(1.30)$ & $0.63(1.39)$ & $0.52(1.24)$ & $0.65(1.25)$ & $0.58(1.43)$ & $0.69(1.51)$ & $0.61(1.3)$ \\
\hline $\begin{array}{l}\text { Patients with outpa- } \\
\text { tient office visit, } \\
n(\%)\end{array}$ & $1542(99.2)$ & $1536(98.8)$ & $869(99.0)$ & $870(99.1)$ & $391(99.2)$ & $386(98.0)$ & $282(100)$ & $280(99.3)$ \\
\hline \multicolumn{9}{|l|}{ MS-related HCRU } \\
\hline $\begin{array}{l}\text { Inpatient admission, } \\
n(\%)\end{array}$ & $125(8.0)$ & $68(4.4)$ & $54(6.2)$ & $38(4.3)$ & $39(9.9)$ & $22(5.6)$ & $32(11.4)$ & $8(2.8)$ \\
\hline LOS, mean (SD) & $7.96(20.56)$ & $11.71(27.07)$ & $10.54(29.99)$ & $12.97(33.19)$ & $3.87(2.69)$ & $4.23(3.54)$ & $8.59(10.57)$ & $26.25(26.92)$ \\
\hline $\begin{array}{l}\text { Patients with ED } \\
\text { visit, } n(\%)\end{array}$ & $298(19.2)$ & $298(19.2)$ & $160(18.2)$ & $155(17.7)$ & $78(19.8)$ & $78(19.8)$ & $60(21.3)$ & $65(23.0)$ \\
\hline $\begin{array}{l}\text { Number of ED visits, } \\
\text { mean (SD) }\end{array}$ & $0.32(0.09)$ & $0.34(1.02)$ & $0.31(0.83)$ & $0.29(0.92)$ & $0.3(0.71)$ & $0.39(1.22)$ & $0.39(1.25)$ & $0.42(1.03)$ \\
\hline $\begin{array}{l}\text { Patients with outpa- } \\
\text { tient office visit, } \\
n(\%)\end{array}$ & $1520(97.8)$ & $1496(96.3)$ & $854(97.3)$ & $841(95.8)$ & $385(97.7)$ & $378(95.9)$ & $281(99.6)$ & 277 (98.2) \\
\hline
\end{tabular}

$E D$ emergency department, $H C R U$ healthcare resource utilization, $L O S$ length of stay, $M S$ multiple sclerosis

\subsection{Subgroup Analysis of Patients Switching from Injectable Disease-Modifying Therapies}

Baseline demographic and clinical characteristics were similar between the overall study population and the subgroup of $1116(71.8 \%)$ patients who switched from an injectable DMT. The proportion of patients who switched from an injectable to an injectable DMT $(n=268)$ and were adherent (45.5\%) was lowest compared with those who switched from an injectable to an oral ( $n=145 ; 56.5 \%$ adherent) or infusion ( $n=172 ; 70.9 \%$ adherent) DMT. Patients who switched from an injectable to an oral DMT had higher persistence $(62.4 \%)$ than those who switched from an injectable to an injectable DMT (48.1\%). The primary reason for nonpersistence was discontinuation.

Patients who switched from an injectable to an oral DMT had the lowest mean all-cause and MS-related total cost of care (\$73,153 and $\$ 62,190$, respectively) compared with those who switched to another injectable $(\$ 78,355$ and $\$ 71,654$, respectively) or an infusion (\$100,313 and $\$ 81,594$, respectively) DMT. Patients who switched to an oral DMT had the lowest increase in mean all-cause total cost of care $(\$ 17,956$ vs. $\$ 20,384$ [injectable DMT] and $\$ 29,248$ [infusion DMT]) and mean MS-related total cost of care $(\$ 15,859$ vs. $\$ 22,229$ [injectable DMT] and $\$ 22,972$ [infusion DMT]). Finally, a decrease in the proportion of patients with all-cause and MS-related inpatient admission or ED visit was observed from pre- to post-index for all patients initiating injectable DMTs, regardless of index DMT route of administration. However, among patients who switched from an injectable to an infusion DMT, there was a greater increase in mean all-cause and MS-related inpatient LOS (13.8 and 29.1 days, respectively) compared with those who switched from an injectable to an oral DMT (0.2 and 4.3 days, respectively) or to another injectable DMT (1.0 and -0.1 days, respectively).

\section{Discussion}

This retrospective, claims-based study is one of the few to analyze treatment patterns, relapse, and economic outcomes in a real-world population of MS patients who switch to a second DMT and to describe the association of route of administration with adherence, persistence, relapse, cost, and HCRU after the switch. One key observation from this study was the high proportion of patients with MS who switched to oral DMTs after initial treatment with an injectable DMT. This study also demonstrated that there are opportunities to improve compliance with DMTs; among all patients who switched, adherence and persistence were suboptimal in the year after the switch 
regardless of the new DMT route of administration. However, patients who switched to oral or infusion DMTs had better adherence and persistence than those who switched to injectable DMTs. Multivariate analyses corroborated these descriptive trends. After adjustment for baseline characteristics, patients who switched to oral or infusion DMTs were more likely to be adherent and persistent than those who switched to injectable DMTs. Research has shown that adherence and persistence are important in the reduction of relapses [14], which may slow the progression of MS.

This study also found that relapses continue to be an issue of concern for those who switch DMT treatments-roughly one of five patients who switched experienced a relapse in the year after the switch. Patients treated with oral agents showed improvements in percentage change in relapse compared with patients treated with injectable or infusion DMTs. Specifically, compared with patients who switched to injectable DMTs, those who switched to oral DMTs had a larger percentage reduction in relapse rate from pre- to post-index. And among patients who had two or three preindex relapses, a higher proportion who switched to oral DMTs were relapse-free in the post-index period compared with those who switched to injectable or infusion DMTs. However, it is important to consider that the difference we observed between oral and infusion DMTs in the proportion of patients who were relapse-free may be related to differences in disease severity that cannot be accounted for using insurance claims since they lack MRI scan results (e.g., patients with more severe MS may be more likely to be switched to an infusion therapy). A previous claims analysis of data from MarketScan research databases from MS patients who switched DMTs reported an increase in the proportion of relapse-free patients post-switch, although approximately one-third of patients still experienced at least one relapse following the switch [15]. Specifically, relapse rates were similar for patients who switched to oral and injectable DMTs (48\% and 45\%, respectively) and highest for those who switched to infusion DMTs (61\%). Multivariate analysis in this claims analysis showed that a patient who switched to an oral DMT had a reduced risk of relapse [15].

Descriptive analysis of cost and HCRU showed a marked increase in cost after switching, primarily driven by the cost of DMTs and other MS-related treatments and services. Although post-index outpatient pharmacy costs were similar across all routes of administration, all-cause total cost of care and medical costs were lowest for patients who switched to oral DMTs, followed by injectable DMTs. Patients who switched to infusion DMTs had the highest post-index costs relative to patients who switched to oral and injectable DMTs. These findings suggest that switching to an oral DMT may confer some cost advantage, in addition to possible improvement in patient adherence and persistence.
Persistence and adherence remained suboptimal across the DMTs that were available during the study period, which pointed to the need for additional treatment options and strategies to enhance compliance. Shared decisionmaking particularly encourages increased patient-centered education, active decision-making, and an individualized approach to treatment, and may lead to increased satisfaction and adherence [24, 25]. Additionally, a technology-based tool is being developed to enhance treatment compliance through the use of mobile and internet applications, which can be used to determine adherence, monitor disease symptoms, and track relevant clinical outcomes [26]. The choice of DMT is a complex decision; although current American Academy of Neurology practice guidelines for patients with MS provide minimal guidance regarding DMT selection, they recommend that clinicians factor in benefit/risk profile, patient preference, and likelihood of adherence when making treatment choices [10]. DMT adherence and persistence are critical components of a clinical management strategy for MS - adherence is associated with improvements in relapse outcomes and quality of life, decreased HCRU and costs, and the slowing of disability progression [12]. Additionally, gaps in therapy of 90 days or longer increase the probability of severe relapse twofold, and severe relapses are associated with substantial increases in healthcare costs $[8,14,27]$. While oral DMTs offer a more convenient route of administration that has the potential to increase rates of adherence compared with injectable DMTs, frequency of dosing, efficacy, and adverse effects are also important factors that impact adherence and persistence [14]. There is a need for development of new oral therapies for MS treatment, and findings herein suggest switching to an oral DMT may reduce relapses and be cost neutral.

Several limitations should be noted. Patients could have switched treatments previously outside our retrospective cohort study timeframe - and so this was the first observed switch only. This study population was limited to those individuals with commercial health coverage or private Medicare supplemental coverage and may not be generalizable to an uninsured population. As is inherent in all administrative claims database analyses, there is a potential for misclassification of MS disease status, clinical and demographic characteristics, and study outcomes; with the use of claims data, disease severity is unknown. Similarly, adherence and persistence were based on filled prescriptions in the outpatient pharmacy setting only and patients were assumed to take medications as prescribed; therefore, data may not reflect whether patients took their therapy as prescribed. Adherence and persistence with medication use cannot be captured in the inpatient or ED setting in a claims database. The DMTs studied in this analysis were limited to US Food and Drug Administration-approved products available during the study period, and DMTs approved after or during 
the study period were not considered. Finally, restricting the study to a 12-month follow-up period could potentially bias the population to patients with less severe disease, since patients who died or went on long-term disability because of serious health conditions could have a follow-up period of less than 12 months.

\section{Conclusion}

This retrospective real-world study examined treatment patterns, relapse, and economic outcomes among patients with MS and a first observed treatment switch in DMT. The findings suggest that switching to an oral DMT may lead to improvement in patient adherence and persistence. However, low adherence, poor persistence, treatment discontinuation, and relapse remain an issue for patients with MS. New treatment options that optimize adherence and persistence while maintaining a favorable risk-benefit profile at comparable cost may improve outcomes and disease management for patients with MS.

Supplementary Information The online version contains supplementary material available at https://doi.org/10.1007/s40801-021-00251-w.

Acknowledgements Support for third-party writing assistance for this manuscript was provided by Samantha Rivera, MS, of Peloton Advantage, LLC (Parsippany, NJ, USA), an OPEN Health company, and funded by Bristol Myers Squibb.

\section{Declarations}

Funding This study was sponsored by Bristol Myers Squibb, Princeton, NJ, USA.

Potential conflicts of interests/Competing interests Leorah Freeman has received consultancy fees from Celgene and Biogen; has received program sponsorship from Biogen and EMD Serono; and has participated on advisory boards for Genentech, Novartis, Celgene, and EMD Serono. Arianna Kee is employed by Bristol Myers Squibb. Marc Tian and Rina Mehta were employed by Celgene, a Bristol Myers Squibb company, at the time the study was conducted.

Ethics approval Not applicable.

Consent to participate Not applicable.

Consent for publication Not applicable

Code availability Not applicable.

Availability of data and material BMS policy on data sharing may be found at https://www.bms.com/researchers-and-partners/independentresearch/data-sharing-request-process.html.

Author contributions All authors were involved in the design and conduction of the study, as well as in drafting the manuscript and revising it critically for intellectual content. All authors had access to the data and interpreted the data and agree to be accountable for all aspects of the work related to the accuracy and integrity of any part of the work. All authors have approved the final version for submission.

Open Access This article is licensed under a Creative Commons Attribution-NonCommercial 4.0 International License, which permits any non-commercial use, sharing, adaptation, distribution and reproduction in any medium or format, as long as you give appropriate credit to the original author(s) and the source, provide a link to the Creative Commons licence, and indicate if changes were made. The images or other third party material in this article are included in the article's Creative Commons licence, unless indicated otherwise in a credit line to the material. If material is not included in the article's Creative Commons licence and your intended use is not permitted by statutory regulation or exceeds the permitted use, you will need to obtain permission directly from the copyright holder. To view a copy of this licence, visit http://creativecommons.org/licenses/by-nc/4.0/.

\section{References}

1. Nicholas JA, Edwards NC, Edwards RA, Dellarole A, Grosso M, Phillips AL. Real-world adherence to, and persistence with, once- and twice-daily oral disease-modifying drugs in patients with multiple sclerosis: a systematic review and meta-analysis. BMC Neurol. 2020;20(1):281.

2. Rieckmann P. Concepts of induction and escalation therapy in multiple sclerosis. J Neurol Sci. 2009;277(Suppl 1):S42-5.

3. Wallin MT, Culpepper WJ, Campbell JD, Nelson LM, LangerGould A, Marrie RA, et al. The prevalence of MS in the United States: a population-based estimate using health claims data. Neurology. 2019;92(10):e1029-40.

4. Hunter SF. Overview and diagnosis of multiple sclerosis. Am J Manag Care. 2016;22(Suppl 6):S141-50.

5. Hirst C, Ingram G, Pearson O, Pickersgill T, Scolding N, Robertson $\mathrm{N}$. Contribution of relapses to disability in multiple sclerosis. J Neurol. 2008;255(2):280-7.

6. Lublin FD, Baier M, Cutter G. Effect of relapses on development of residual deficit in multiple sclerosis. Neurology. 2003;61(11):1528-32.

7. Ma VY, Chan L, Carruthers KJ. Incidence, prevalence, costs, and impact on disability of common conditions requiring rehabilitation in the United States: stroke, spinal cord injury, traumatic brain injury, multiple sclerosis, osteoarthritis, rheumatoid arthritis, limb loss, and back pain. Arch Phys Med Rehabil. 2014;95(5):986-95. e1.

8. Parise H, Laliberte F, Lefebvre P, Duh MS, Kim E, Agashivala N, et al. Direct and indirect cost burden associated with multiple sclerosis relapses: excess costs of persons with MS and their spouse caregivers. J Neurol Sci. 2013;330(1-2):71-7.

9. Owens GM. Economic burden of multiple sclerosis and the role of managed care organizations in multiple sclerosis management. Am J Manag Care. 2016;22(6 Suppl):s151-8.

10. Rae-Grant A, Day GS, Marrie RA, Rabinstein A, Cree BAC, Gronseth GS, et al. Practice guideline recommendations summary: disease-modifying therapies for adults with multiple sclerosis: report of the Guideline Development, Dissemination, and Implementation Subcommittee of the American Academy of Neurology. Neurology. 2018;90(17):777-88.

11. Finkelsztejn A. Multiple sclerosis: overview of disease-modifying agents. Perspect Medicin Chem. 2014;6:65-72.

12. Burks J, Marshall TS, Ye X. Adherence to disease-modifying therapies and its impact on relapse, health resource utilization, and costs among patients with multiple sclerosis. ClinicoEcon Outcomes Res. 2017;9:251-60. 
13. Goldschmidt C, McGinley MP. Advances in the treatment of multiple sclerosis. Neurol Clin. 2021;39(1):21-33.

14. Patti F. Optimizing the benefit of multiple sclerosis therapy: the importance of treatment adherence. Patient Prefer Adherence. 2010;4:1-9.

15. Bowen J, Mehta R, Pelletier C, Tian M, Noxon V, Johnson BH, et al. Treatment patterns among patients with multiple sclerosis initiating second-line disease-modifying therapy. Adv Ther. 2020;37(7):3163-77.

16. Le Page E, Edan G. Induction or escalation therapy for patients with multiple sclerosis? Rev Neurol (Paris). 2018;174(6):449-57.

17. Desai RJ, Mahesri M, Gagne JJ, Hurley E, Tong A, Chitnis T, et al. Utilization patterns of oral disease-modifying drugs in commercially insured patients with multiple sclerosis. J Manag Care Spec Pharm. 2019;25(1):113-21.

18. Johnson KM, Zhou H, Lin F, Ko JJ, Herrera V. Real-world adherence and persistence to oral disease-modifying therapies in multiple sclerosis patients over 1 year. J Manag Care Spec Pharm. 2017;23(8):844-52.

19. Setayeshgar S, Kingwell E, Zhu F, Zhang T, Carruthers R, Marrie RA, et al. Persistence and adherence to the new oral diseasemodifying therapies for multiple sclerosis: a population-based study. Mult Scler Relat Disord. 2019;27:364-9.

20. Cree BAC, Mares J, Hartung HP. Current therapeutic landscape in multiple sclerosis: an evolving treatment paradigm. Curr Opin Neurol. 2019;32(3):365-77.
21. Culpepper WJ, Marrie RA, Langer-Gould A, Wallin MT, Campbell JD, Nelson LM, et al. Validation of an algorithm for identifying MS cases in administrative health claims datasets. Neurology. 2019;92(10):e1016-28.

22. Ollendorf DA, Jilinskaia E, Oleen-Burkey M. Clinical and economic impact of glatiramer acetate versus beta interferon therapy among patients with multiple sclerosis in a managed care population. J Manag Care Pharm. 2002;8(6):469-76.

23. Chastek BJ, Oleen-Burkey M, Lopez-Bresnahan MV. Medical chart validation of an algorithm for identifying multiple sclerosis relapse in healthcare claims. J Med Econ. 2010;13(4):618-25.

24. Tintoré M, Alexander M, Costello K, Duddy M, Jones DE, Law $\mathrm{N}$, et al. The state of multiple sclerosis: current insight into the patient/health care provider relationship, treatment challenges, and satisfaction. Patient Prefer Adherence. 2017;11:33-45.

25. Ben-Zacharia A, Adamson M, Boyd A, Hardeman P, Smrtka J, Walker B, et al. Impact of shared decision making on diseasemodifying drug adherence in multiple sclerosis. Int J MS Care. 2018;20(6):287-97.

26. Pratap A, Grant D, Vegesna A, Tummalacherla M, Cohan S, Deshpande $\mathrm{C}$, et al. Evaluating the utility of smartphone-based sensor assessments in persons with multiple sclerosis in the real-world using an app (elevateMS): observational, prospective pilot digital health study. JMIR mHealth uHealth. 2020;8(10):e22108.

27. O'Connell K, Kelly SB, Fogarty E, Duggan M, Buckley L, Hutchinson $\mathrm{M}$, et al. Economic costs associated with an MS relapse. Mult Scler Relat Disord. 2014;3(6):678-83. 